\title{
Full characterization and modeling of graded interfaces in a high lattice-mismatch axial nanowire heterostructure
}

\author{
D. V. Beznasyuk $\odot,{ }^{1}$ P. Stepanov, ${ }^{1}$ J. L. Rouvière $\odot,{ }^{2}$ F. Glas $\odot,{ }^{3}$ M. Verheijen $\odot,{ }^{4,5}$ J. Claudon $\odot,{ }^{2}$ and M. Hocevar $\odot^{1, *}$ \\ ${ }^{1}$ Université Grenoble-Alpes, CNRS-Institut Néel, 25 av. des Martyrs, 38000 Grenoble, France \\ ${ }^{2}$ Université Grenoble-Alpes, CEA-IRIG-PHELIQS, 17 av. des Martyrs, 38000 Grenoble, France \\ ${ }^{3}$ Centre for Nanoscience and Nanotechnology, Université Paris-Saclay, CNRS, 10 bd. Thomas Gobert, 91120 Palaiseau, France \\ ${ }^{4}$ Eurofins Materials Science Netherlands, High Tech Campus 11, 5656AE Eindhoven, The Netherlands \\ ${ }^{5}$ Department of Applied Physics, Eindhoven University of Technology, 5600 MB Eindhoven, The Netherlands
}

(Received 5 December 2019; accepted 15 June 2020; published xxxxxxxxxx)

\begin{abstract}
Controlling the strain level in nanowire heterostructures is critical for obtaining coherent interfaces of high crystalline quality and for the setting of functional properties such as photon emission, carrier mobility, or piezoelectricity. In a nanowire axial heterostructure featuring a sharp interface, strain is set by the materials lattice mismatch and the nanowire radius. Here we show that introducing a graded interface in nanowire heterostructures offers an additional parameter to control strain. For a given interface length and lattice mismatch, we first derive theoretically the maximum nanowire radius below which coherent growth is possible. We validate these findings by growing and characterizing various $\mathrm{In}(\mathrm{Ga}) \mathrm{As} / \mathrm{GaAs}$ nanowire heterostructures with graded interfaces. We perform chemical and structural characterization of the interface by combining energy-dispersive $\mathrm{x}$-ray spectroscopy and high resolution transmission electron microscopy. In the case of coherent growth, we observe that the mismatch strain relaxes elastically on the side walls of the nanowire around the interface area, while the core of the nanowire remains partially strained. Our experimental data show good agreement with finite element calculations. In particular, this analysis confirms that mechanical strain is largely reduced by interface grading. Overall, our work extends the parameter space for the design of nanowire heterostructures, thus opening new opportunities for nanowire optoelectronics.
\end{abstract}

DOI: 10.1103/PhysRevMaterials.00.004600

\section{INTRODUCTION}

Semiconductor nanowires offer the unique opportunity to realize coherent axial heterostructures which associate materials having vastly different lattice parameters [1-3] or crystalline structures $[4,5]$. In addition, the nanowire geometry can be adjusted to finely engineer its photonic and electronic properties [6-10]. Brought together, these appealing features promise a wealth of applications in optoelectronics $[6,10]$. Prototypes of laser diodes [10,11] and quantum light sources [12], white light emitting diodes [13,14], solar cells [7-9], and high efficiency photodetectors $[15,16]$ were recently developed in nanowire heterostructures.

Today, numerous material combinations have been explored to realize axial nanowire heterostructures [4,17-19]. In all cases, the control of the strain level around the interface is critical, because above a certain threshold, elastic energy is plastically released via the formation of dislocations $[20,21]$. Dislocations act as recombination centers for photons and electrons and degrade the materials properties by reducing the light emission or detection efficiency, and the carrier density. One solution to realize defect-free interfaces is to reduce the nanowire lateral dimensions. Indeed, for a given couple of materials A and B, and thus a given lattice mismatch, there exists a critical nanowire radius below which coherent growth of B on top of A is possible regardless of the height of B.

*Corresponding author: moira.hocevar@neel.cnrs.fr
The mismatch strain is then partially and elastically relaxed at the nanowire sidewalls. This critical radius, which separates the domains of elastic and plastic strain relaxation, is well understood in the case of a sharp interface [20,22].

However, sharp interfaces still present a large residual strain, which may be detrimental for applications. For example, the electron-hole wave-function overlap decreases in InAs/GaAs quantum dot nanowires, resulting in longer exciton lifetimes and nonradiative recombination [23]. High interface strains also lead to potential barriers for charge carriers, which limits their transport in quantum dot nanowire devices [24]. Moreover, a high interfacial strain can enhance piezoelectric effects which degrade performances of nanowire based solar cells [25]. Finally, in the case of large lattice mismatch, coherent growth is only possible for a very limited range of radii, severely limiting the accessible geometries. As an example, for a lattice mismatch of $7 \%$, the critical radius is as low as $10 \mathrm{~nm}$. Implementing graded interfaces, with a smooth chemical profile, offers a solution to overcome these limitations. Despite a few works [25-27], this strategy remains to be thoroughly explored.

In this work we investigate both theoretically and experimentally graded interfaces in axial nanowire heterostructures. A theoretical model specifies the wire radii compatible with coherent growth for various interface lengths and lattice mismatches. While we specifically consider the representative case of interfaces whose chemical profile is described by an error function, these calculations could be easily extended to 
other profiles. We compare these predictions to experiments realized with the highly mismatched InAs/GaAs material system. The nanowire heterostructures are grown by molecular beam epitaxy (MBE), using a gold droplet as a catalyst. We perform a complete characterization of the interface: the chemical profile is obtained by energy dispersive $\mathrm{x}$-ray spectroscopy (EDX) analysis, while the structural characterization is conducted through high-resolution transmission electron microscopy (TEM). In the case of coherent growth, the maps of the mismatch strain obtained by geometrical phase analysis (GPA) are in excellent agreement with finite element simulations. This analysis confirms in particular that mechanical strain is largely reduced by interface grading. More generally, interface grading constitutes a novel tuning knob to adjust the physical properties of nanowire heterostructures.

\section{RESULTS AND DISCUSSION}

As schematized in Fig. 1(a), we consider an infinitely long nanowire oriented along the $z$ direction, with a circular section of radius $R$. The nanowire features a graded interface between two materials A and B. They have different lattice parameters $a$, which leads to the lattice mismatch $\varepsilon_{m}=\left(a_{B}-a_{A}\right) / a_{A}$. The interface is centered at $z=0$, and the fractions $n_{A}$ and $n_{B}=1-n_{A}$ of the two species follow a smooth profile. For small-scale compositional gradients (on the order of the nanowire diameter), the interface chemical profile is usually well represented by an error function [28-30]. Specifically, we assume:

$$
n_{B}=\frac{1}{2}\left[1+\operatorname{erf}\left(\frac{z}{L}\right)\right] \text { with } \operatorname{erf}\left(\frac{z}{L}\right)=\frac{2}{\sqrt{\pi}} \int_{0}^{\frac{z}{L}} e^{-u^{2}} d u
$$

where $L$ measures the interface length [Fig. 1(b)]. For a given couple of materials, and in the framework of linear elasticity, the amplitude of the strain generated around the interface is controlled by the normalized interface length $\alpha=L / R$. Intuitively, one thus expects that the critical radius $R_{c}$ below which coherent growth is possible increases with $\alpha$. To determine $R_{c}$ for a given lattice mismatch and interface profile, we compare the energies of the system in two states, namely state (1) with a purely elastic relaxation of the mechanical strain, and state (2) with a single dislocation segment lying perpendicular to the nanowire axis. The critical radius is then defined as the radius above which state (2) has an energy lower than state (1). In state (1), the system energy reduces to elastic energy. This quantity is evaluated with finite element software, assuming mechanical isotropy for the materials. The energy in state (2) is obtained with the method of Spencer and Tersoff considering an edge dislocation [31,32]. More details on these calculations are given in Sec. S1 of the Supplemental Material [33]. Figure 1(c) gives the variation of the critical radius $R_{c}$ as a function of mismatch $\varepsilon_{m}$, each curve corresponding to a given normalized interface length $\alpha$. Alternatively, the curves can be read as giving the critical mismatch (below which the system should remain coherent) as a function of nanowire radius. They thus separate, in the $R-\varepsilon_{m}$ plane, the domains of elastic/plastic (below/above) growth. The case $\alpha=0$ corresponds to a sharp interface. Strikingly, increasing $\alpha$ leads to a dramatic increase in $R_{c}$. (a) (b)
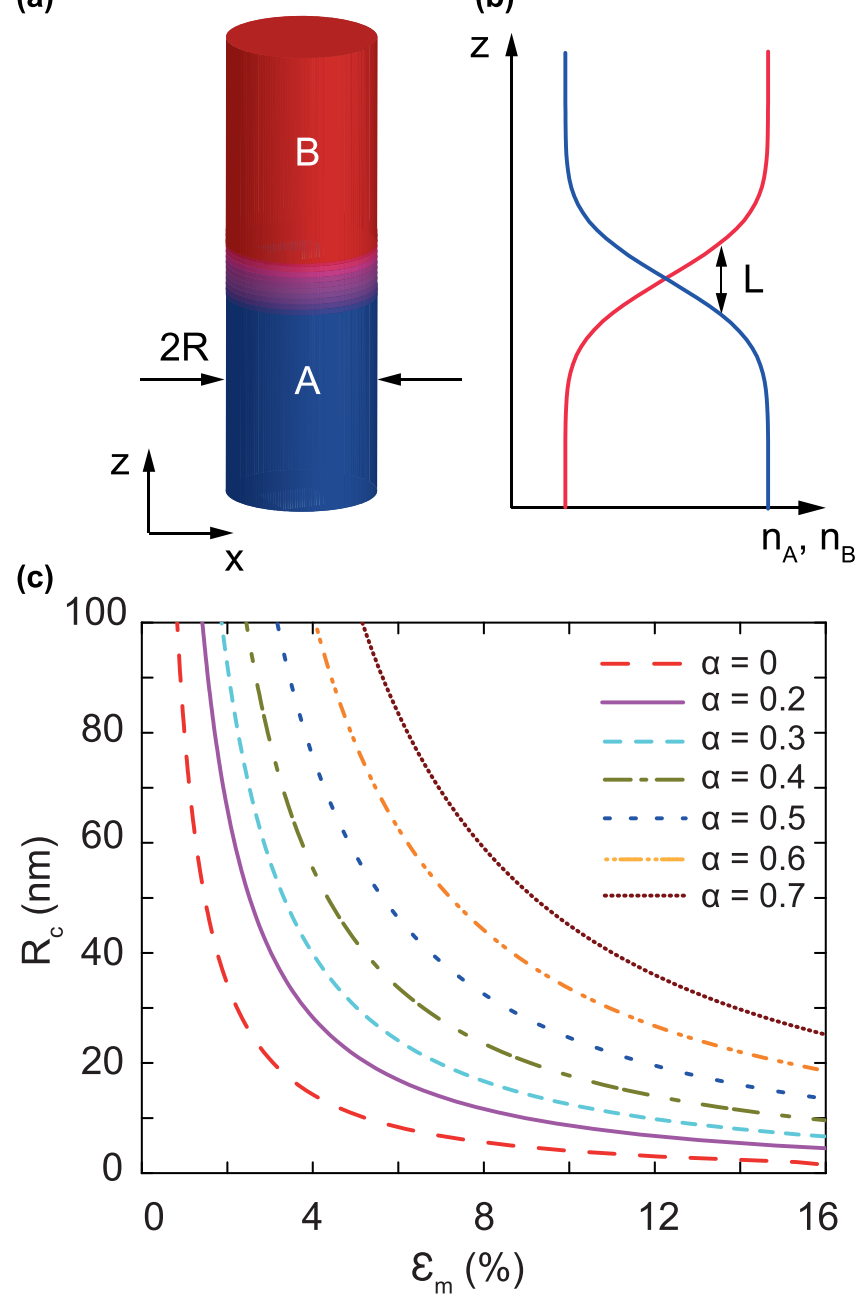

FIG. 1. Axial nanowire heterostructure with graded interfaces. (a) Schematics of a nanowire heterostructure composed of two materials $\mathrm{A}$ and $\mathrm{B}$. The nanowire features a circular section (radius $R$ ), its longitudinal axis coincides with the $z$ direction. (b) Composition profile along the nanowire axis described by an error function [Eq. (1)]. Over the interface length $L$, the composition varies by $52 \%$ of the total composition jump. (c) Calculated critical radius $R_{c}$ as a function of the mismatch strain $\varepsilon_{m}$ between $\mathrm{A}$ and $\mathrm{B}$ for different values of $\alpha=L / R$ ( $\alpha=0$ corresponds to an abrupt interface).

Composition-graded interfaces thus considerably extend the domain where coherent growth is possible: an interface length over tens of nanometers is sufficient to completely suppress the constraint on the nanowire dimensions. This is in contrast to thin film epitaxy [34] where composition-graded buffer layers need to be larger than hundreds of nanometers and to selective area growth of planar nanowires where compositiongraded interfaces of tens of nanometers are not sufficient to release the mechanical strain and suppress misfit dislocations at the interface [35]. These results, obtained with the parameters of the InAs/GaAs material system, capture the general benefits of interface grading. Furthermore, they can be used to estimate $R_{c}$ for graded interfaces involving other material systems. When precise values are required, one can employ the same method to determine $R_{c}$ using the specific 
(a)
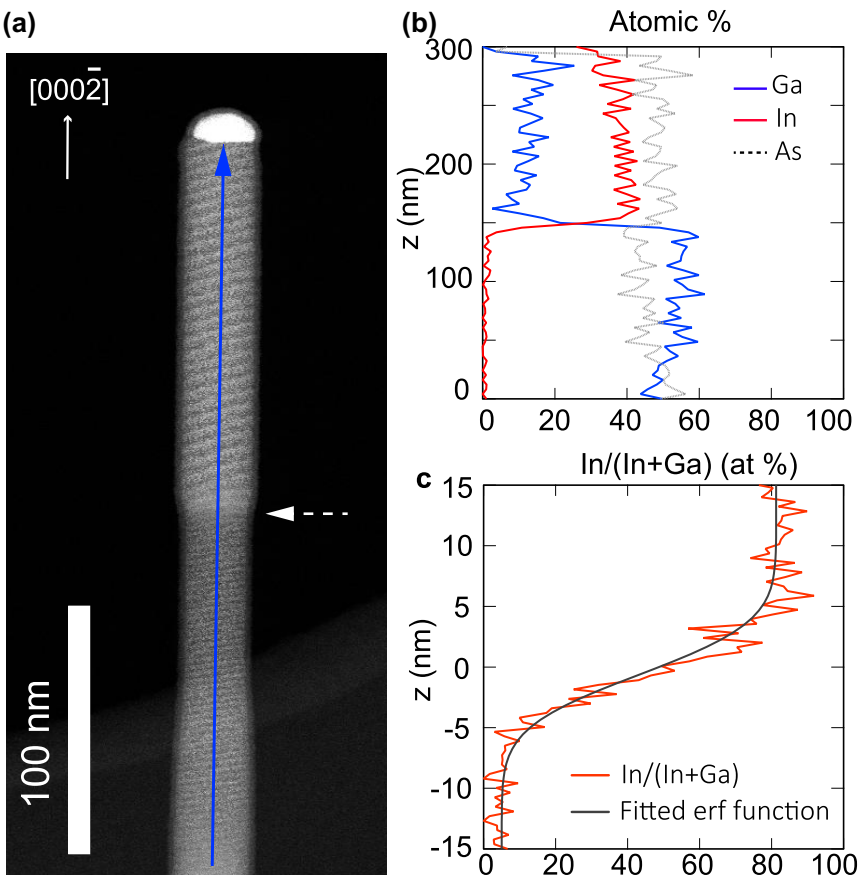

FIG. 2. InGaAs/GaAs axial nanowire heterostructure: chemical characterization. (a) Dark-field TEM image of $\mathrm{NW}_{1}$ taken along the [2-1-10] zone axis. The position of the interface is indicated by the white arrow. Moiré fringes are visible in the nanowire and are due to the coincidence periods between the scanning step of the electron beam and the interatomic potential. (b) EDX composition profile measured along the nanowire axis [blue arrow in (a)]. (c) Zoom on the interface profile. The fit to an error function yields $L=5.9 \mathrm{~nm}$.

mechanical properties of the materials as well as the actual composition profile of the interface.

In the following we investigate experimentally axial nanowire heterostructures in the $\operatorname{In}(\mathrm{Ga}) \mathrm{As} / \mathrm{GaAs}$ material system in order to compare the theoretical predictions for the evolution of $R_{c}$ versus $\alpha$ with experimental data sets. Interfaces with $\varepsilon_{m}$ varying from $0 \%$ to $7 \%$ can be fabricated in the $\operatorname{In}(\mathrm{Ga}) \mathrm{As} / \mathrm{GaAs}$ material system thanks to the possibility of creating ternary alloys. We focus here on high-mismatch heterostructures with $\varepsilon_{m} \geqslant 5.7 \%$. We grow our $\operatorname{In}(\mathrm{Ga}) \mathrm{As} / \mathrm{GaAs}$ nanowire heterostructures by MBE using the gold assisted vapor-liquid-solid mechanism [36]. The nanowire radius is controlled by the catalyst dimensions, while the interface length can be controlled by adjusting the growth conditions. Indeed, interface grading occurs in particle-seeded nanowire systems and is attributed to the solubility of the growth species in the liquid droplet which constitutes a reservoir [37]. This "reservoir effect" can be tuned or suppressed by carefully adjusting the growth parameters and the droplet dimensions to form either sharp or controlled graded interfaces [38-40]. In this work we have grown several nanowire heterostructures with different $R, \varepsilon_{m}$, and $\alpha$ (Supplemental Material S2). In all cases we performed a structural and chemical characterization of the interface which we detail for a first sample labeled $\mathrm{NW}_{1}$.

Figure 2(a) shows an image of $\mathrm{NW}_{1}$, obtained by scanning transmission electron microscopy using the high-angle annular dark-field imaging mode (HAADF-STEM). The HAADF-
STEM image yields a nanowire radius of $10.5 \mathrm{~nm}$ and suggests that the position of the interface stands right after the bottleneck visible in Fig. 2(a). This is confirmed by the energy dispersive $\mathrm{x}$-ray spectroscopy (EDX) line profile measured along the nanowire axis [Fig. 2(b)]. The bottom segment is composed of pure GaAs and the upper one is made of an InGaAs ternary alloy with an average indium composition of 0.8 . The corresponding lattice mismatch is $\varepsilon_{m}=5.7 \%$ (wurtzite $a$ lattice parameter). Note that EDX radial profiles across both the InGaAs segment and the top of the GaAs segment do not reveal any radial shell. As shown in Fig. 2(c), the chemical profile of the interface is very well reproduced by an error function. The fit of the data to Eq. (1) leads to an interface length $L$ of $5.9 \mathrm{~nm}$ [Fig. 2(c)] which corresponds to a reduced interface length $\alpha=0.56$. The nanowire radius lies well below the critical value $R_{c}=56 \mathrm{~nm}$, calculated from the experimental value of $\alpha$ and $\varepsilon_{m}$ [Fig. 1(c)].

To investigate the crystalline quality of the nanowire heterostructure, we image different areas of $\mathrm{NW}_{1}$ by high resolution HAADF STEM followed by fast Fourier transform. Both GaAs and InGaAs segments have the wurtzite (WZ) crystal structure except for a small zinc blende (ZB) insertion in the interface region (Supplemental Material S3). Figures 3(a) and 3(b) are additional HR STEM images in two different orientations and do not reveal any misfit dislocation in the crystal at the InGaAs/GaAs interface. As predicted by our calculations, the crystalline integrity of our nanowire is preserved and the mismatch strain at the interface is relaxed elastically.

Across the interface, the lattice parameters $a$ and $c$ are modified both by compositional changes and by mechanical strain. We employ geometric phase analysis (GPA) to image the $c$ - and $a$-lattice strain, i.e., the $c$ - and $a$-lattice deformations with respect to a reference chosen here as unstrained $c$-GaAs and $a$-GaAs: $\frac{\Delta c}{c}=\frac{c-c_{\mathrm{GAAs}}}{c_{\mathrm{GAAs}}}$ and $\frac{\Delta a}{a}=\frac{a-a_{\mathrm{GaAs}}}{a_{\mathrm{GaAs}}}$, respectively [41]. To map the $c$-lattice (a-lattice) strain around the interface, we use the high resolution [2 $\overline{1} \overline{1} 0]$ [01 10$]$ HAADFSTEM image shown in Fig. 3(a) [Fig. 3(b)]. GPA is then performed to the image by applying a mask around the $(000 \overline{2})$ ( $2 \overline{1} \overline{1} 0)$ Bragg peak in the Fourier transform visible in the inset of Fig. 3(a) [Fig. 3(b)] (Supplemental Material S3). We choose a medium-size mask of $\sim 0.15|\vec{g}|$ (with $\vec{g}$ the reciprocal lattice vector) in order to preserve a balance between a good spatial resolution and a high signal-to-noise ratio $[42,43]$.

Figure 3(c) [Fig. 3(d)] shows the resulting color-coded map of $\Delta c / c(\Delta a / a)$ in the $a-c$ plane. The bottom part of the wire corresponds to unstrained GaAs $(\Delta c / c=\Delta a / a=0 \%)$. The top part of the nanowire features a maximum deformation of the $c$ and $a$ planes with respect to GaAs which is consistent with unstrained wurtzite $\operatorname{In}_{0.8} \mathrm{Ga}_{0.2} \mathrm{As}$ (composition found by EDX), which indicates full relaxation far from the interface. We observe a transition region around the $\mathrm{InGaAs} / \mathrm{GaAs}$ interface indicating that the lattice is gradually stretched. Importantly, there is no discontinuity (or defects) in the transition regions for $\Delta c / c$ and $\Delta a / a$, confirming the absence of misfit dislocations at the interface $[44,45]$. The transition region is thicker in the center than on the nanowire edges, showing that the $a$ - and $c$-lattice parameters recover faster their unstrained characteristic value near the nanowire sidewalls than at the nanowire center. It is indeed more difficult to release strain in the core of the nanowire than on the free sidewalls. 

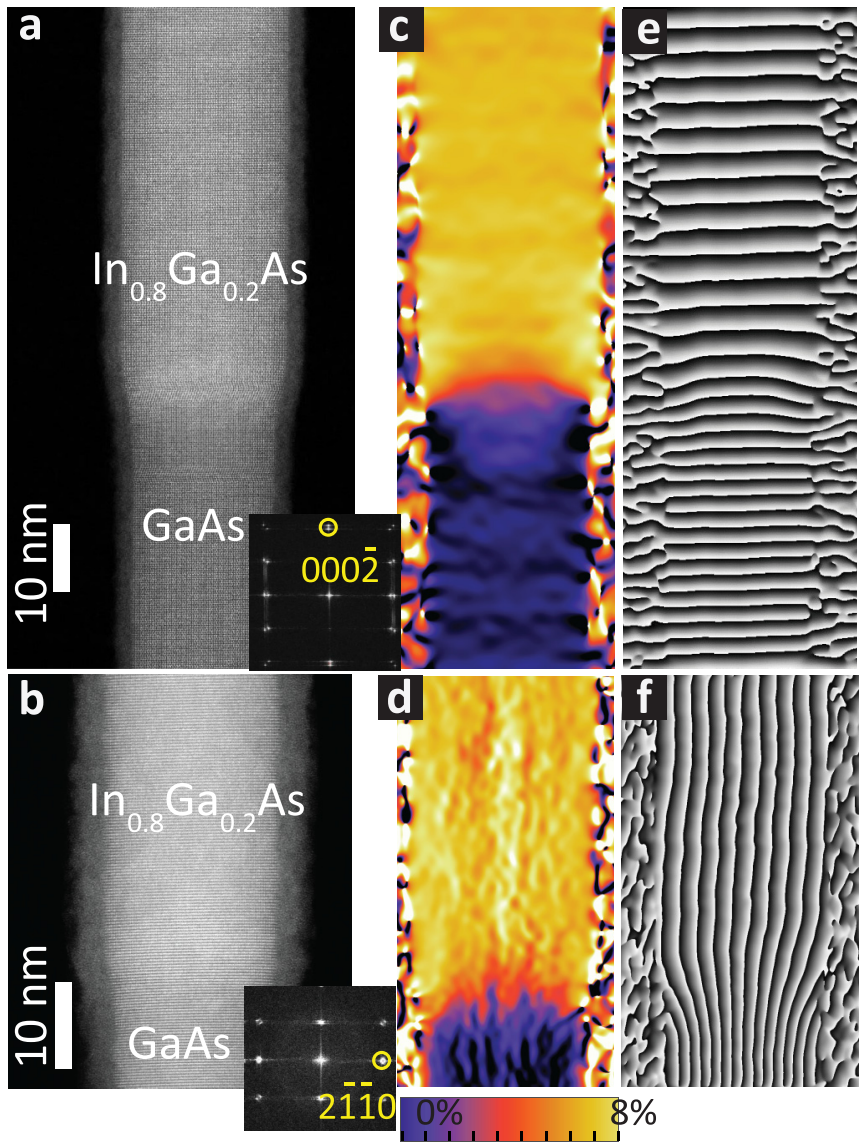

FIG. 3. InGaAs/GaAs axial nanowire heterostructure: high resolution structural characterization. HAADF STEM image taken along the [2-1-10] viewing direction (a) and the [01-10] viewing direction (b). The insets show the corresponding fast Fourier transform (FFT). Map of the mismatch strain $\Delta c / c$ (c) and $\Delta a / a$ (d) obtained by applying GPA on (a) and (b), respectively. Corresponding numerical moiré patterns (e) and (f). The scale bars are identical for (a), (c), and (e). Similarly, the scale bars are identical for (b), (d), and (f).

To get a complementary insight on strain relaxation, we vi- sualize the arrangement of the crystal planes with a numerical moiré technique [46] [Figs. 3(e) and 3(f)]. We obtain a moiré pattern from the geometric phase images of Figs. 3(a) and 3(b) using Fourier filtering of the $(000 \overline{2})$ and $(2 \overline{1} \overline{1} 0)$ Bragg peaks, respectively. We observe that the distance between planes is larger in the upper segment than in the bottom segment. Far from the interface, the planes are parallel to each other and are strain-free. Near the InGaAs/GaAs interface, at the sidewalls, the planes bend dramatically. This large deformation is due to elastic relaxation of the mismatch strain at the nanowire free surfaces. Note that plane bending is also evidenced in lattice rotations maps obtained by GPA (not shown).

We now quantitatively compare the experimental GPA data to numerical simulations. We first calculate the mechanical strain tensor $\overline{\bar{\varepsilon}}$ around the nanowire interface using finite element software (COMSOL Multiphysics), the values of lattice constants $[47,48]$ and stiffness coefficients [49] for WZ InAs and WZ GaAs, and all the measured characteristics of $\mathrm{NW}_{1}$. We consider a cylindrical wire of radius $R=10.5 \mathrm{~nm}$. The lengths of the GaAs and InGaAs sections (200 and
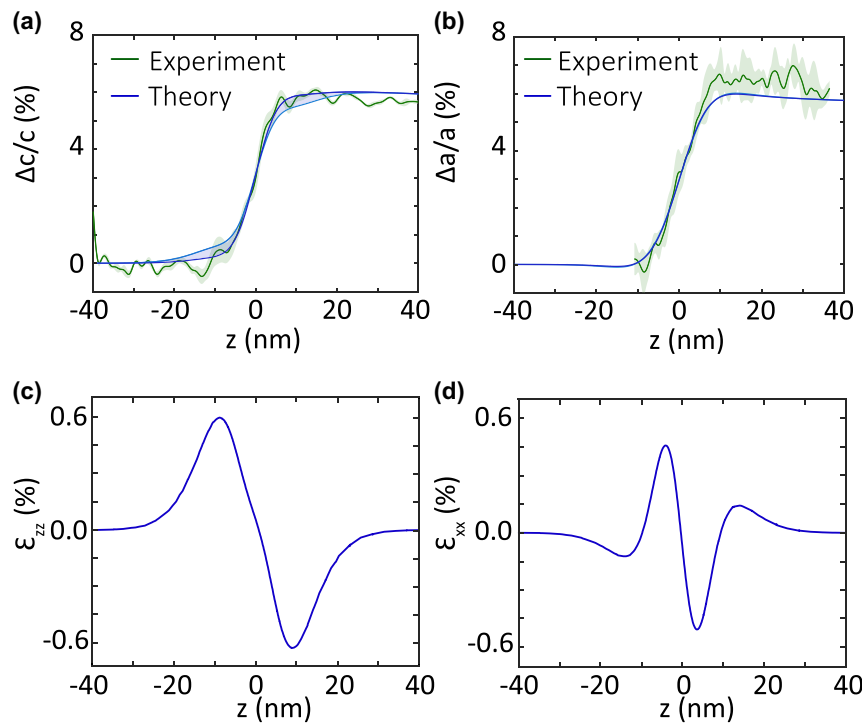

FIG. 4. Experimental and simulated strain profiles along the $\mathrm{z}$ axis. Evolution of $\Delta c / c$ (a) and $\Delta a / a$ (b) across the interface. The experimental GPA data profiles (green, with standard deviation as error bars) were extracted from the strain maps and averaged over a lateral sampling of 80 pixels. The simulated profiles were extracted in the central part of the nanowire. They correspond to calculated data along the nanowire axis only (blue) and to calculated data averaged along the nanowire depth (purple). Evolution of the calculated mechanical strain components $\varepsilon_{\mathrm{zz}}$ (c) and $\varepsilon_{\mathrm{xx}}$ (d) across the interface.

$60 \mathrm{~nm}$, respectively) correspond to the dimensions of $\mathrm{NW}_{1}$. Since these lengths are already both much larger than $R$, the results will also apply to nanowires featuring longer segments. We also include the interface chemical profile as determined from the fit to the EDX measurement [Fig. 2(b)]. Finally, we take into account the mechanical anisotropy associated with the wurtzite nanowire crystal (see the Supplemental Material S4 Methods). The $a$-lattice strain is then deduced using the relation $\Delta a / a=\left[a_{\mathrm{loc}}\left(\varepsilon_{x x}+1\right)-a_{\mathrm{GaAs}}\right] / a_{\mathrm{GaAs}}$. Here $a_{\mathrm{loc}}$ is the local unstrained lattice parameter, determined from the measured chemical profile in Fig. 2(c) and using a linear interpolation between GaAs and InAs, $a_{\mathrm{GaAs}}$ is the unstrained lattice parameter of GaAs and $\varepsilon_{x x}=\frac{a-a_{\mathrm{loc}}}{a_{\mathrm{loc}}}$ is the mechanical strain along the $x$ axis. Similarly, we have $\Delta c / c=$ $\left[c_{\mathrm{loc}}\left(\varepsilon_{z z}+1\right)-c_{\mathrm{GaAs}}\right] / c_{\mathrm{GaAs}}$. In addition, in order to account for the depth of focus of STEM imaging (around $10 \mathrm{~nm}$ ), the theoretical data are averaged along the nanowire depth (details in the Supplemental Material S4).

Figure 4(a) and 4(b) compare the experimental and simulated $a$ - and $c$-lattice strain along the nanowire axis. Without any free parameter, we obtain for $\Delta c / c$ an excellent agreement between the simulated and experimental profiles (the discrepancy is lower than $0.5 \%$ ). For $\Delta a / a$, the agreement is good, but the theory predicts a slightly lower In composition in the $\mathrm{In}_{0.8} \mathrm{Ga}_{0.2}$ As segment than observed in the experimental data. We attribute this to the noise in the experimental GPA data [Fig. 3(d)], which increases the uncertainty on the unstrained reference region. Both $\Delta c / c$ and $\Delta a / a$ increase gradually from $0 \%$ (GaAs reference) to about $5.9 \%$ and $5.7 \%$ $\left(\mathrm{In}_{0.8} \mathrm{Ga}_{0.2} \mathrm{As}\right.$ segment) respectively, which is consistent with 


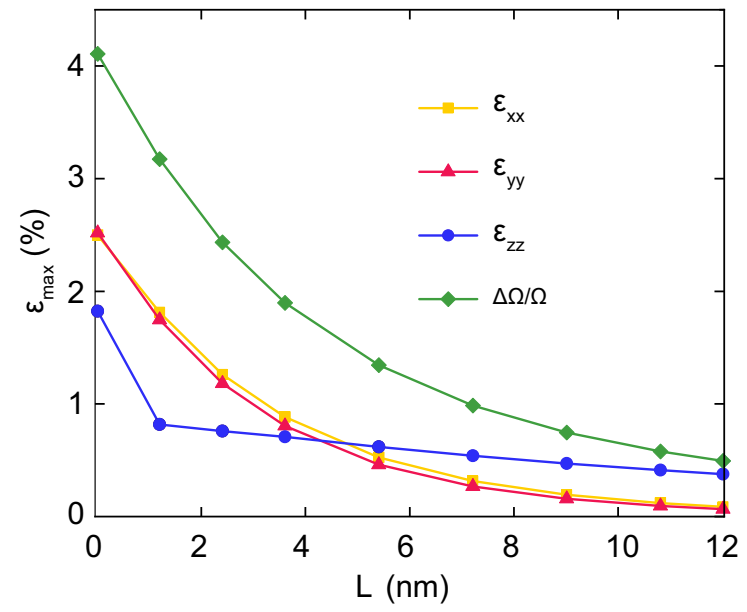

FIG. 5. Influence of the interface length on the mechanical strain. The maximum hydrostatic strain $\Delta \Omega / \Omega$ as well as the maxima of the mechanical strain components $\varepsilon_{x x}, \varepsilon_{y y}$, and $\varepsilon_{z z}$ are plotted versus the interface length $L$.

the calculated lattice mismatch with the WZ phase. Furthermore, we also compute theoretical 2D maps of $\Delta c / c$ and $\Delta a / a$ (Supplemental Material S4). They both reproduce the features observed in the experimental maps. In particular, the different radial strain profiles extracted from the GPA maps at different $z$ show that the InGaAs segment experiences an increase in the $c$-lattice strain on the side walls with respect to the center of the segment, while the GaAs segment sees a decrease in the $c$-lattice strain. These data follow the simulated radial strain profiles (Supplemental Material S4). Overall, this demonstrates that we have a quantitative understanding of the structural properties of the interface.

We build on this understanding to discuss the distribution of mechanical strain around the InGaAs/GaAs interface. Figures 4(c) and 4(d) show the calculated strain components $\varepsilon_{z z}$ and $\varepsilon_{x x}$ along the nanowire axis $(z)$. Both components are zero far from the interface, and feature significant amplitude over a domain which is $30-40 \mathrm{~nm}$ long. Its size significantly exceeds the interface length $(L=5.9 \mathrm{~nm})$, and is in fact roughly set by the nanowire diameter, in agreement with the Saint Venant's principle. $\varepsilon_{z z}$ and $\varepsilon_{x x}$ show a maximum around $0.5 \%$, indicating that the mismatch strain is largely decreased but not fully released. Finally, both $\varepsilon_{z z}$ and $\varepsilon_{x x}$ feature large spatial inhomogeneities. In particular, $\varepsilon_{x x}$ presents several longitudinal oscillations between tensile and compressive deformation. Importantly, these marked strain inhomogeneities will introduce a spatial modulation of the band structure $[23,24]$ which should be taken into account in the design of nanowire devices.

Figure 5 illustrates the dramatic influence of interface grading on the strain fields. We consider an InGaAs/GaAs nanowire with the same dimensions and composition as $\mathrm{NW}_{1}$ and plot the maximal values of $\varepsilon_{x x}, \varepsilon_{\mathrm{yy}}$, and $\varepsilon_{z z}$ as a function of the interface length $L$. In the case of a sharp interface $(L=0) \varepsilon_{z z}$ and $\varepsilon_{x x}$ reach $1.7 \%$ and $2.6 \%$, respectively. A graded interface with $L=5.9 \mathrm{~nm}\left(\mathrm{NW}_{1}\right)$ is already sufficient to decrease $\varepsilon_{z z}$ by a factor of 3 , and $\varepsilon_{x x}$ by a factor close to 6 . We note here that interface grading has a stronger

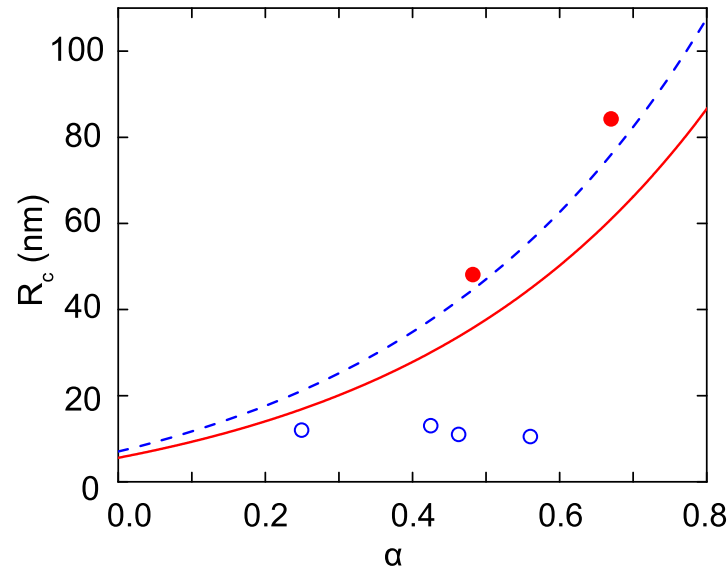

FIG. 6. Elastic and plastic relaxation in axial nanowire heterostructures. The elastic and plastic domains are separated by a line corresponding to the calculated $R_{c}$ for $\operatorname{In}_{0.8} \mathrm{Ga}_{0.2} \mathrm{As} / \mathrm{GaAs}$ (dashed blue) and InAs/GaAs (solid red). Above the line, theory predicts plastic relaxation. The circles indicate the experimental data from dislocation-free (open) and plastically relaxed (solid) structures.

influence on the transverse strain components. Of course, increasing $L$ leads to a further decrease of the strain but for the investigated interface lengths, the spatial extension of the strained region is roughly the same (Supplemental Material S4). We next consider the hydrostatic strain $\Delta \Omega / \Omega=\varepsilon_{x x}+$ $\varepsilon_{y y}+\varepsilon_{z z}$, which has an important impact on the band gap and the conduction band offsets [23]. Its maximum value is also plotted in Fig. 5: it is reduced from $4.2 \%$ down to $0.8 \%$ as the interface length increases from 0 to $8.4 \mathrm{~nm}$. Modest interface grading thus already results in a strong reduction of the strain level.

Next, we consider additional nanowire samples to further support the theoretical predictions of the coherent growth domains. The results are summarized in Fig. 6, which confronts the theory to experimental results obtained with two families of samples. The first set of nanowires $\left(\mathrm{NW}_{2}\right.$ to $\left.\mathrm{NW}_{4}\right)$ features $\varepsilon_{m}$ of $5.7 \%, R$ around $10 \mathrm{~nm}$, and $\alpha$ ranging from 0.25 to 0.56 . For all these nanowires, the mismatch strain is always elastically released by the sidewalls, as shown on HRTEM images by the absence of dislocations at the interface and on GPA color-coded maps by the curvature of the $a$ and $c$ planes (Supplemental Material S5). As shown in Fig. 6, this nanowire family falls in the predicted coherent domain. The second set of InAs/GaAs nanowire samples with $\alpha$ being 0.48 and 0.67 for a unique $\varepsilon_{m}$ of $7.1 \%\left(\mathrm{NW}_{5}\right.$ and $\left.\mathrm{NW}_{6}\right)$. We observe by HRTEM that the nanowires present defects at the InAs/GaAs interface. GPA color-coded maps confirm the presence of misfit dislocations and reveal plane bending (Supplemental Material S6). In those nanowires the mismatch strain is released both via plastic and elastic relaxation. We finally plot the experimental data in Fig. 6: these thick nanowires fall in the plastic relaxation region, confirming here as well the predictions.

Our study is of particular significance when it comes to realize optoelectronic devices using semiconductor heterostructures. Material combinations such as, for example, $\operatorname{InP} / \operatorname{InSb}\left(\varepsilon_{m}=10 \%\right)$ and $\mathrm{GaN} / \mathrm{InN}\left(\varepsilon_{m}=11 \%\right)$ are 
important for photovoltaic and optoelectronic applications but their structural quality and therefore their physical functions suffer from an extremely high lattice mismatch. As seen in previous works, reducing the diameter is not always possible or sufficient to prevent plastic relaxation [44,50]. Thus, the design of nanowire devices with compositionally graded interfaces has the potential to reduce materials constraint on the device dimensions. Importantly, a compositional grading over few nanometers at nanowire interfaces is sufficient to reduce most of the strain without altering the required physical properties.

\section{CONCLUSION}

In conclusion, we fully characterized high lattice-mismatch axial $\operatorname{In}(\mathrm{Ga}) \mathrm{As} / \mathrm{GaAs}$ heterostructure nanowires featuring graded interfaces. The heterostructure shows a preserved crystalline quality with a mismatch strain released elastically, via plane bending. Full elastic relaxation occurs at the nanowire sidewalls while the remaining strain is localized in the cen- tral area of the nanowire, larger than the interface length. Theoretical predictions confirmed by our experimental data show that the domains for coherent growth can be extended using compositional gradients of few nanometers. Beyond the realization of coherent heterointerfaces, interface grading offers an additional tuning knob to control residual strain in the nanowire, and thus to fine-tune its optoelectronic properties.

\section{ACKNOWLEDGMENTS}

Assistance with the MBE growth by Yann Genuist is gratefully acknowledged. Martien den Hertog, Eric Robin, and Joël Cibert are acknowledged for fruitful discussions. The authors acknowledge financial support by the LABEX LANEF (ANR-10-LABX-51-01), Université Grenoble Alpes (program AGIR), the Thomas Jefferson Fund of the Embassy of France in the United States and the FACE Foundation, as well as the ANR HYBRID (ANR-17-PIRE-0001) and QDOT (ANR-16-CE09-0010-01).

The authors declare no conflict of interest.

[1] N. Jegenyes, M. Morassi, P. Chrétien, L. Travers, L. Lu, F. Julien, M. Tchernycheva, F. Houzé, and N. Gogneau, High piezoelectric conversion properties of axial InGaN/GaN nanowires, Nanomaterials 8, 367 (2018).

[2] P. Caroff, M. E. Messing, M. Borg, B. K. Dick, K. Deppert, and L.-E. Wernersson, InSb heterostructure nanowires: MOVPE growth under extreme lattice mismatch, Nanotechnology 20, 495606 (2009).

[3] C.-Y. Wen, M. C. Reuter, J. Bruley, J. Tersoff, S. Kodambaka, E. Stach, and F. M. Ross, Formation of compositionally abrupt axial heterojunctions in silicon-germanium nanowires, Science 326, 1247 (2009).

[4] M. Hocevar, G. Immink, M. Verheijen, N. Akopian, V. Zwiller, L. Kouwenhoven, and E. Bakkers, Growth and optical properties of axial hybrid III-V/silicon nanowires, Nat. Commun. 3, 1266 (2012).

[5] D. L. Dheeraj, G. Patriarche, H. Zhou, T. B. Hoang, A. F. Moses, S. Grønsberg, A. T. J. van Helvoort, B.-O. Fimland, and H. Weman, Growth and characterization of wurtzite GaAs nanowires with defect-free zinc blende GaAsSb inserts, Nano Lett.8, 4459 (2008).

[6] Y. Zhang, J. Wu, M. Aagesen, and H. Liu, III-V nanowires and nanowire optoelectronic devices, J. Phys. D. Appl. Phys. 48, 463001 (2015).

[7] P. Krogstrup, H. I. Jørgensen, M. Heiss, O. Demichel, J. V. Holm, M. Aagesen, J. Nygard, and A. F. Morral, Singlenanowire solar cells beyond the Shockley-Queisser limit, Nat. Photon. 7, 306 (2013).

[8] L. Wen, Z. Zhao, X. Li, Y. Shen, H. Guo, and Y. Wang, Theoretical analysis and modeling of light trapping in high efficicency GaAs nanowire array solar cells, Appl. Phys. Lett. 99, 143116 (2011).

[9] L. Hu and G. Chen, Analysis of optical absorption in silicon nanowire arrays for photovoltaic applications, Nano Lett. 7, 3249 (2007).

[10] L. N. Quan, J. Kang, C.-Z. Ning, and P. Yang, Nanowires for photonics, Chem. Rev. 119, 9153 (2019).

[11] S. W. Eaton, A. Fu, A. B. Wong, C.-Z. Ning, and P. Yang, Semiconductor nanowire lasers, Nat. Rev. Mater. 1, 16028 (2016).

[12] M. E. Reimer, G. Bulgarini, N. Akopian, M. Hocevar, M. B. Bavinck, M. a. Verheijen, E. P. A. M. Bakkers, L. P. Kouwenhoven, and V. Zwiller, Bright single-photon sources in bottom-up tailored nanowires, Nat. Commun. 3, 737 (2012).

[13] S. M. Sadaf, Y.-H. Ra, H. P. T. Nguyen, M. Djavid, and Z. Mi, Alternating-current InGaN/GaN tunnel junction nanowire white-light emitting diodes, Nano Lett. 15, 6696 (2015).

[14] H. P. T. Nguyen, M. Djavid, S. Y. Woo, X. Liu, A. T. Connie, S. Sadaf, Q. Wang, G. a. Botton, I. Shih, and Z. Mi, Engineering the carrier dynamics of InGaN nanowire white light-emitting diodes by distributed p-AlGaN electron blocking layers, Sci. Rep. 5, 7744 (2015).

[15] S. J. Gibson, B. van Kasteren, B. Tekcan, Y. Cui, D. van Dam, J. E. M. Haverkort, E. P. A. M. Bakkers, and M. E. Reimer, Tapered InP nanowire arrays for efficient broadband high-speed single-photon detection, Nat. Nanotechnol. 14, 473 (2019).

[16] R. R. Lapierre, M. Robson, K. M. Azizur-Rahman, and P. Kuyanov, A review of III-V nanowire infrared photodetectors and sensors, J. Phys. D. Appl. Phys. 50, 123001 (2017).

[17] J. V Holm, H. I. Jørgensen, P. Krogstrup, J. Nygård, H. Liu, and M. Aagesen, Surface-passivated GaAsP single-nanowire solar cells exceeding $10 \%$ efficiency grown on silicon, Nat. Commun. 4, 1498 (2013).

[18] E. P. A. M. Bakkers, M. T. Borgström, and M. A. Verheijen, Epitaxial growth of III-V nanowires on group IV substrates, MRS Bull. 32, 117 (2007).

[19] E. Barrigón, M. Heurlin, Z. Bi, B. Monemar, and L. Samuelson, Synthesis and applications of III-V nanowires, Chem. Rev. 119 9170 (2019).

[20] F. Glas, Critical dimensions for the plastic relaxation of strained axial heterostructures in free-standing nanowires, Phys. Rev. B 74, 121302 (2006).

[21] K. L. Kavanagh, Misfit dislocations in nanowire heterostructures, Semicond. Sci. Technol. 25, 24006 (2010). 
[22] M. W. Larsson, J. B. Wagner, M. Wallin, P. Håkansson, L. E. Fröberg, L. Samuelson, and L. R. Wallenberg, Strain mapping in free-standing heterostructured wurtzite InAs/InP nanowires, Nanotechnology 18, 15504 (2006).

[23] Y. M. Niquet, Electronic properties of InAs/GaAs nanowire superlattices, Phys. E Low-dimensional Syst. Nanostruct. 37, 204 (2007).

[24] Y.-M. Niquet and D. Mojica, Quantum dots and tunnel barriers in InAs/InP nanowire heterostructures: Electronic and optical properties, Phys. Rev. B 77, 115316 (2008).

[25] A. T. M. Golam Sarwar and R. C. Myers, Exploiting piezoelectric charge for high performance graded $\mathrm{InGaN}$ nanowire solar cells, Appl. Phys. Lett. 101, 143905 (2012).

[26] N. Erhard, A. T. M. G. Sarwar, F. Yang, D. W. McComb, R. C. Myers, and A. W. Holleitner, Optical control of internal electric fields in band gap-graded InGaN nanowires, Nano Lett. 15, 332 (2014).

[27] H. Zhang, H. Han, S. Xiong, H. Wang, S. Volz, and Y. $\mathrm{Ni}$, Impeded thermal transport in composition graded $\mathrm{SiGe}$ Nanowires, Appl. Phys. Lett. 111, 121907 (2017).

[28] T. E. Schlesinger and T. Kuech, Determination of the Interdiffusion of $\mathrm{Al}$ and $\mathrm{Ga}$ in Undoped (Al,Ga)As/GaAs Quantum wells, Appl. Phys. Lett. 49, 519 (1986).

[29] D. Goren, G. Asa, and Y. Nemirovsky, Barrier formation at graded $\mathrm{HgTe} / \mathrm{CdTe}$ heterojunctions, J. Appl. Phys. 80, 5083 (1996).

[30] H. Han, A. Beyer, J. Belz, A. König, W. Stolz, and K. Volz, Quantitative atomic resolution at interfaces: Subtraction of the background in STEM images with the example of (Ga, In) P/GaAs structures, J. Appl. Phys. 121, 25301 (2017).

[31] B. J. Spencer and J. Tersoff, Stresses and first-order dislocation energetics in equilibrium Stranski-Krastanow islands, Phys. Rev. B 63, 205424 (2001).

[32] R. Gatti, A. Marzegalli, V. A. Zinovyev, F. Montalenti, and L. Miglio, Modeling the plastic relaxation onset in realistic SiGe islands on Si(001), Phys. Rev. B 78, 184104 (2008).

[33] See Supplemental Material at http://link.aps.org/supplemental/ 10.1103/PhysRevMaterials.xx.xxxxxx for details on the critical diameter calculations, nanowire growth, TEM and GPA analysis, mechanical simulations, simulated and experimental 2D strain maps, GPA analysis of additional defect-free nanowires, and of dislocated interfaces.

[34] J. Zou, D. J. H. Cockayne, and B. F. Usher, Misfit dislocations and critical thickness in InGaAs/GaAs heterostructure systems, J. Appl. Phys. 73, 619 (1993).

[35] M. Friedl, K. Cerveny, P. Weigele, G. Tütüncüoglu, S. MartíSánchez, C. Huang, T. Patlatiuk, H. Potts, Z. Sun, M. O. Hill et al., Template-assisted scalable nanowire networks, Nano Lett. 18, 2666 (2018).

[36] D. V. Beznasyuk, E. Robin, M. D. Hertog, J. Claudon, and M. Hocevar, Dislocation-free axial InAs-on-GaAs nanowires on silicon, Nanotechnology 28, 365602 (2017).
[37] N. Li, T.Y. Tan, and U. Gösele, Transition region width of nanowire hetero-and pn-junctions grown using vapor-liquidsolid processes, Appl. Phys. A 90, 591 (2008).

[38] K. A. Dick, J. Bolinsson, B. M. Borg, and J. Johansson, Controlling the abruptness of axial heterojunctions in III-V nanowires: Beyond the reservoir effect, Nano Lett. 12, 3200 (2012).

[39] G. Priante, F. Glas, G. Patriarche, K. Pantzas, F. Oehler, and J.-C. Harmand, Sharpening the interfaces of axial heterostructures in self-catalyzed AlGaAs nanowires: Experiment and theory, Nano Lett. 16, 1917 (2016).

[40] V. G. Dubrovskii, Understanding the vapor-liquid-solid growth and composition of ternary III-V nanowires and nanowire heterostructures, J. Phys. D. Appl. Phys. 50, 453001 (2017).

[41] D. Cooper, T. Denneulin, N. Bernier, A. Béché, and J.-L. Rouvière, Strain mapping of semiconductor specimens with $\mathrm{nm}$-scale resolution in a transmission electron microscope, Micron 80, 145 (2016).

[42] M. Vallet, Y. Claveau, B. Warot-Fonrose, C. Gatel, J. Nicolai, N. Combe, C. Magen, R. Teissier, A. N. Baranov, and A. Ponchet, Highly strained AlAs-type interfaces in InAs/AlSb heterostructures, Appl. Phys. Lett. 108, 211908 (2016).

[43] J. Li, C. Zhao, Y. Xing, S. Su, and B. Cheng, Full-field strain mapping at a $\mathrm{Ge} / \mathrm{Si}$ heterostructure interface, Materials (Basel) 6, 2130 (2013).

[44] C. Frigeri, D. Scarpellini, A. Fedorov, S. Bietti, C. Somaschini, V. Grillo, L. Esposito, M. Salvalaglio, A. Marzegalli, and F. S. Montalenti, Interface abruptness and strain relaxation in selfassisted grown InAs/GaAs nanowires, Appl. Surf. Sci. 395, 29 (2017).

[45] M. de la Mata, C. Magén, P. Caroff, and J. Arbiol, Atomic scale strain relaxation in axial semiconductor III-V nanowire heterostructures, Nano Lett. 14, 6614 (2014).

[46] M. J. Hÿtch, J.-L. Putaux, and J.-M. Pénisson, Measurement of the displacement field of dislocations to $0.03 \AA$ by electron microscopy, Nature (London) 423, 270 (2003).

[47] D. Kriegner, C. Panse, B. Mandl, K. A. Dick, M. Keplinger, J. M. Persson, P. Caroff, D. Ercolani, L. Sorba, F. Bechstedt et al., Unit cell structure of crystal polytypes in InAs and InSb nanowires, Nano Lett.11, 1483 (2011).

[48] D. Jacobsson, F. Yang, K. Hillerich, F. Lenrick, S. Lehmann, D. Kriegner, J. Stangl, L. R. Wallenberg, K. A. Dick, and J. Johansson, Phase transformation in radially merged wurtzite GaAs nanowires, Cryst. Growth Design 15, 4795 (2015).

[49] S. Q. Wang and H. Q. Ye, First-principles study on elastic properties and phase stability of III-V compounds, Phys. Status Solidi 240, 45 (2003).

[50] A. Pitanti, D. Ercolani, L. Sorba, S. Roddaro, F. Beltram, L. Nasi, G. Salviati, and A. Tredicucci, InAs/InP/InSb Nanowires as Low Capacitance $n-n$ Heterojunction Diodes, Phys. Rev. X 1, 011006 (2011). 